\title{
An enhanced correlation identification algorithm and its application on spread spectrum induced polarization data
}

\author{
Siming $\mathrm{He}^{1,2}$, Jian Guan ${ }^{3}$, Xiu Ji ${ }^{1,4}$, Hang $\mathrm{Xu}^{1}$, and Yi Wang ${ }^{2}$ \\ ${ }^{1}$ School of Electrical and Information Engineering, Changchun Institute of Technology, Changchun 130000, China \\ ${ }^{2}$ College of Instrumentation and Electrical Engineering, Jilin University, Changchun 130000, China \\ ${ }^{3}$ College of Electronic Science and Engineering, Jilin University, Changchun 130000, China \\ ${ }^{4}$ National Local Joint Engineering Research Center for Smart Distribution Grid Measurement and Control with Safety \\ Operation Technology, Changchun Institute of Technology, Changchun 130000, China
}

Correspondence: Yi Wang (wangyijlu@jlu.edu.cn) and Xiu Ji (jixiu523@163.com)

Received: 29 March 2020 - Discussion started: 26 June 2020

Revised: 20 March 2021 - Accepted: 6 April 2021 - Published: 19 May 2021

\begin{abstract}
In spread spectrum induced polarization (SSIP) data processing, attenuation of background noise from the observed data is the essential step that improves the signalto-noise ratio (SNR) of SSIP data. The time-domain spectral induced polarization based on pseudorandom sequence (TSIP) algorithm has been proposed to improve the SNR of these data. However, signal processing in background noise is still a challenging problem. We propose an enhanced correlation identification (ECI) algorithm to attenuate the background noise. In this algorithm, the cross-correlation matching method is helpful for the extraction of useful components of the raw SSIP data and suppression of background noise. Then the frequency-domain IP (FDIP) method is used for extracting the frequency response of the observation system. Experiments on both synthetic and real SSIP data show that the ECI algorithm will not only suppress the background noise but also better preserve the valid information of the raw SSIP data to display the actual location and shape of adjacent high-resistivity anomalies, which can improve subsequent steps in SSIP data processing and imaging.
\end{abstract}

\section{Introduction}

Induced polarization (IP) technology operated in both the time domain and the frequency domain is useful in exploration for groundwater mapping, mineral exploration, and other environmental studies (Revil et al., 2012, 2019; Høyer et al., 2018). Since the phenomenon of IP in the time domain was first discovered by Liu et al. (2017b), there has been consistent efforts to explore its utilization in various research efforts. In 1959, the frequency-domain IP (FDIP) approach was proposed by Collett et al. (1959) and Seigel (1959), which became a classic, widely used mapping technique. For example, the first variable-frequency approach was proposed by Wait (1959), then the spectrum approach of the complex resistivity was developed by Zonge and Wynn (1975), and the dual-frequency IP approach was presented and developed by He (1993) and Han et al. (2013). Recently, spread spectrum induced polarization (SSIP) is a popular branch of FDIP which uses pseudorandom current pulses of opposite polarity as an excitation source (Chen et al., 2007; Xi et al., 2013, 2014; He et al., 2015). According to the intrinsic broadband characteristics of the source itself, the spectral response of an observation system can be simultaneously calculated at multiple frequencies in electrical exploration (Liu et al., 2019). Thus, this SSIP technology has been gaining attention and application in electrical prospecting (Xi et al., 2014; Lu et al., 2019; Wang and He, 2020).

In field detection experiments, it is still a major problem that IP data are often contaminated with background noise. The background noise can be mainly categorized into two types: the Gaussian noise and the impulsive interference with different percentage of outliers (Liu et al., 2016; Kimiaefar et al., 2018; Li et al., 2019). If the background noise is not effectively reduced, the remnant noise can affect the calculation of complex resistivity and may mislead subsequent interpretations of the subsurface structure. 
The field of FDIP denoising has achieved quite good results through the constant research of experts and scholars. There have been many algorithms that can be used to suppress the FDIP random noise (Mo et al., 2017), such as smooth filter (Guo, 2017), Mean stack (Liu, 2015), digital filter (Meng et al., 2015), and robust stacking (Liu et al., 2016). The smooth can effectively attenuate Gaussian noise, but the impulsive interference with intense energy leaves the effectiveness of this algorithm limited. Therefore, an effective attenuating algorithm for background noise is still a challenging task for traditional noise suppression algorithms (Neelamani et al., 2008; Liu et al., 2017a). SSIP method also faces the same issue (Liu and Chen, 2016; Liu et al., 2017b).

Recently, the new algorithm based on a circular crosscorrelation method, time-domain spectral induced polarization based on pseudorandom sequence (TSIP) algorithm, has also been used to suppress the SSIP noise ( $\mathrm{Li}$ et al. 2013; Zhang et al., 2020). Due to its effective denoising ability, the identification method has gained more attention and development. However, the TSIP algorithm is restricted because the excitation signal is sensitive to the random noise. For this problem, we propose an enhanced correlation identification (ECI) algorithm for reducing the noise in SSIP data. The ECI algorithm obtains cross-correlations between the transmitter output signal, the excitation signal, and the response signal. The performance of the ECI algorithm is demonstrated on both synthetic and field SSIP data. Experimental results show that the ECI algorithm can effectively control the root mean square of noise (NRMS) increase, enhance its denoising performance in background noise and improve the valid signal preservation to display the actual location and shape of highresistivity anomalies with higher resolution.

\section{Theory}

\subsection{The TSIP theoretical model}

Figure 1 shows a traditional diagram of the electrical resistivity survey. The transmitter output signal $u_{T}(t)$ is poured from electrode $\mathrm{A}$ to electrode $\mathrm{B}$, the excitation signal $i(t)$ flows from electrode $\mathrm{A}$ to electrode $\mathrm{B}$, and the response signal $u(t)$ between the electrodes $\mathrm{M}$ and $\mathrm{N}$ is measured. To simultaneously obtain the spectral response of subsurface at various frequencies, pseudorandom sequence based the excitation signal $i(t)$ is considered. Thus, the spectral response of subsurface be retrieved by the TSIP algorithm, and its spectral response be expressed as (Li et al., 2013):

$H_{\mathrm{e}}(\omega)=\frac{P_{u i}(\omega)}{P_{i i}(\omega) \cdot P_{\mathrm{S}}(\omega)}$,

where $P_{u i}(\omega)$ is the cross-power spectral density of $u(t)$ and $i(t), P_{i i}(\omega)$ the auto-power spectral density of $i(t)$, and $P_{\mathrm{S}}(\omega)$ is the impulse spectral response of the observing system.
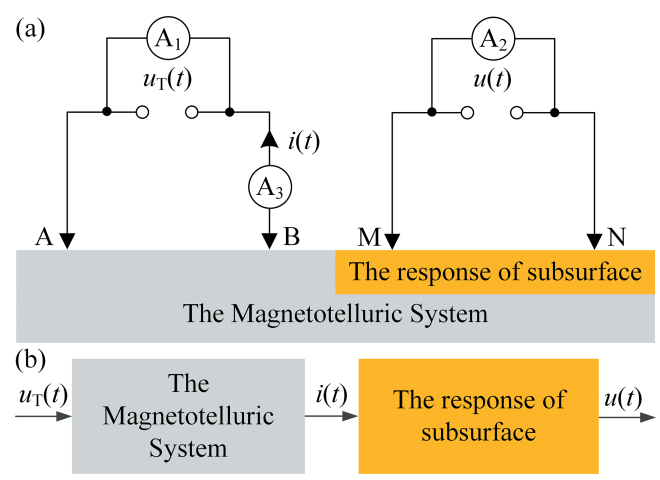

Figure 1. (a) The observation model of the four-electrode measurement. (b) Its equivalent diagram.

Given this observation mode using low-power signals, the magnetotelluric system is a time-invariant system and let us suppose that $H_{\mathrm{S}}(\omega)$ is 1 . Equation (1) can further be expressed as

$H_{\mathrm{e}}(\omega)=\frac{P_{u i}(\omega)}{P_{i i}(\omega)}=\frac{\mathrm{fft}\left[R_{u i}(\tau)\right]}{\operatorname{fft}\left[R_{i i}(\tau)\right]}=\frac{U(\omega)}{I(\omega)}$,

where fft[.] denotes fast Fourier transform (FFT), $R_{u i}(\tau)$ is the cross-correlation function of $u(t)$ and $i(t), R_{i i}(\tau)$ is the autocorrelation function of $i(t), U(\omega)$ and $I(\omega)$ depict the geometric factor defined by the frequency spectrum of $u(t)$ and the frequency spectrum of $i(t)$ respectively, and $\tau$ denotes time delay.

In the practical field environment, this observation mode is contaminated by the background noise, as shown in Fig. 2. The output of the sensors $A_{k}(k=1,2,3)$ can be expressed as

$$
\begin{aligned}
& y_{1}=u_{T}(t)+n_{1}(t), \\
& y_{2}=u(t)+n_{2}(t), \\
& y_{3}=i(t)+n_{3}(t),
\end{aligned}
$$

where $n_{k}(t)$ is the background noise.

Therefore, according to Eq. (2), the formula of the TSIP algorithm is given as

$$
\begin{aligned}
H_{\mathrm{e}}(\omega) & =\frac{P_{y_{2} y_{3}}(\omega)}{P_{y_{3} y_{3}}(\omega)}=\frac{\mathrm{fft}\left[R_{y_{2} y_{3}}(\tau)\right]}{\mathrm{fft}\left[R_{y_{3} y_{3}}(\tau)\right]} \\
& =\frac{\mathrm{fft}\left[R_{u i}(\tau)+R_{u n_{2}}(\tau)+R_{i n_{1}}(\tau)\right]}{\mathrm{fft}\left[R_{i i}(\tau)+R_{i n_{1}}(\tau)+R_{n_{1} n_{1}}(\tau)\right]} \\
& \approx \frac{\mathrm{fft}\left[R_{u i}(\tau)\right]}{\operatorname{fft}\left[R_{i i}(\tau)+R_{n_{3} n_{3}}(\tau)\right]} .
\end{aligned}
$$

Equation (6) demonstrates that the TSIP algorithm has a weak denoising effect when $n_{3}(t)$ is the massive intense noise. In other words, the TSIP algorithm depends on the energy intensity of $n_{3}(t)$ present in $i(t)$. 


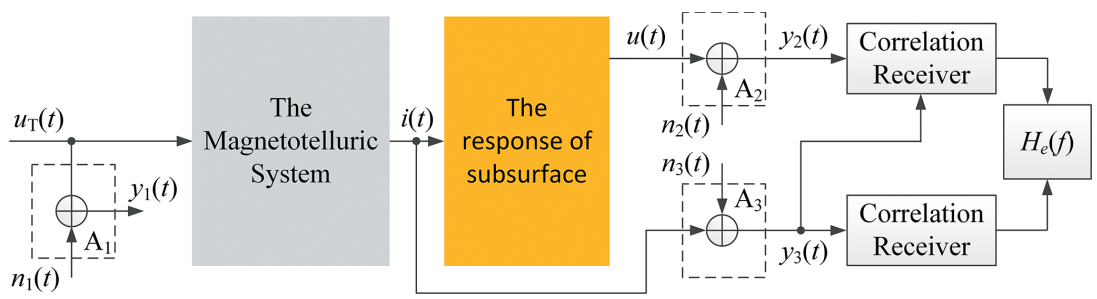

Figure 2. Schematic diagram using the TSIP algorithm.

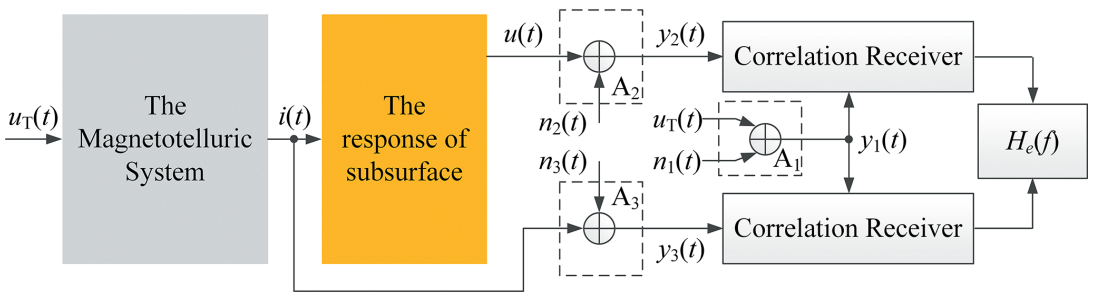

Figure 3. The schematic diagram of the ECI denoising model.

\subsection{The ECI theoretical model}

That the denoising ability of the TSIP algorithm is limited is caused by that $i(t)$ is sensitive to $n_{3}(t)$. To solve this problem, the ECI algorithm is proposed in Fig. 3 and its derivation process is as follows.

Firstly, let us suppose that the telluric system is a timeinvariant system under low-power signals. For three sensor output signals, their cross-correlation functions are the periodic correlation functions of time $\tau$. When the length of the correlation window NT is specified, $0.0125 \mathrm{~s}$ in this experiment. The cross-correlation functions can be expressed as follows:

$$
\begin{aligned}
& R_{y_{1} y_{2}}(\tau)=E\left[y_{1}(t) y_{2}(t-\tau)\right]=\left(R_{u_{T} u}(\tau)\right)_{N}+R_{n_{1} n_{2}}(\tau), \\
& R_{y_{1} y_{3}}(\tau)=E\left[y_{1}(t) y_{3}(t-\tau)\right]=\left(R_{u_{T} i}(\tau)\right)_{N}+R_{n_{1} n_{3}}(\tau)
\end{aligned}
$$

where $R_{n_{1} n_{2}}(\tau)$ and $R_{n_{1} n_{3}}(\tau)$ are the cross-correlations of, $n_{2}(t)$ and $n_{3}(t)$ respectively, and $\tau$ is time delay that lies in the range of $-\mathrm{NT}$ to NT.

Figure 4 shows the schematic diagram of the ZW-CMDSII instrument (Zhang et al., 2014; He et al., 2014). As is known from the figure, we can conclude that $u_{T}(t)$ is mainly disturbed by the floor noise energy of the instrument, and $i(t)$ and $u(t)$ are mainly contaminated by environmental noise. The floor noise is relatively very low, while environmental noise possesses a much higher energy level. Thus we assume that $n_{1}(t) \approx 0$, and can conclude that zero correlation between $n_{1}(t)$ and $n_{2}(t), n_{3}(t), R_{n_{1} n_{2}}(\tau) \approx 0$ and $R_{n_{1} n_{3}}(\tau) \approx$ 0 .

Based on the above analyses, we can further obtain:

$R_{y_{1} y_{2}}(\tau) \approx\left(R_{u_{T} u}(\tau)\right)_{N}$,

$R_{y_{1} y_{3}}(\tau) \approx\left(R_{u_{T} i}(\tau)\right)_{N}$.

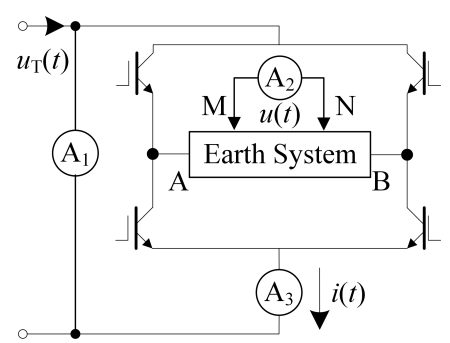

Figure 4. Schematic diagram of the instrument.

Then the cross-power spectrum of Eqs. (9) and (10) can be written as following

$P_{y_{1} y_{2}}(\omega) \approx P_{u_{T} u}(\omega)$

$P_{y_{1} y_{3}}(\omega) \approx P_{u_{T} i}(\omega)$.

Finally, according to Eqs. (2) and (11), Eq. (12) can be expressed as following

$$
\begin{aligned}
H_{\mathrm{e}}(\omega) & =\frac{U(\omega)}{I(\omega)}=\frac{U(\omega) U_{T}^{*}(\omega)}{I(\omega) U_{T}^{*}(\omega)}=\frac{P_{u_{T} u}(\omega)}{P_{u_{T} i}(\omega)} \\
& \approx \frac{P_{y_{1} y_{2}}(\omega)}{P_{y_{1} y_{3}}(\omega)}=\left|\frac{P_{y_{1} y_{2}}(\omega)}{P_{y_{1} y_{3}}(\omega)}\right| e^{-j\left(\varphi_{y 1}(\omega)-\varphi_{y 1}(\omega)\right)}
\end{aligned}
$$

where $\varphi_{y 1} y_{2}(\omega)$ and $\varphi_{y 1 y 3}(\omega)$ denotes the difference between $y_{1}(t), y_{2}(t)$ and $y_{3}(t)$.

So, Eq. (13) is the formula of the ECI algorithm. The derivation process of this formula clearly describes that the ECI algorithm can effectively suppress the background noise and be independent on the degree of $n_{3}(t)$ present in $i(t)$. 
(a)

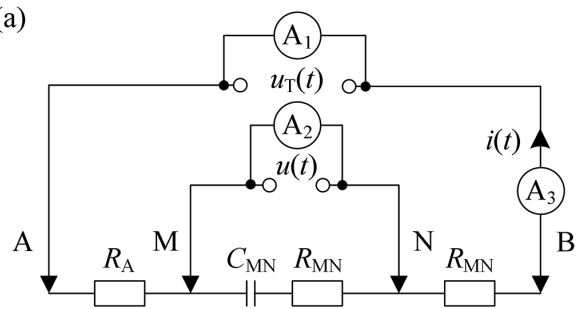

(b)

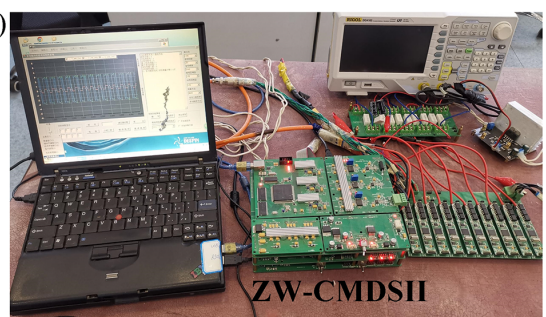

Figure 5. (a) Experimental schematic; (b) experimental setup.

\section{Experiment on synthetic SSIP data record}

We test the ECI algorithm for attenuating background noise of SSIP data sets in comparison with the FDIP algorithm and the TSIP algorithm. For the comparison, the signal-to-noise ratio (SNR), root mean square of noise (NRMS), and relative error $(\varepsilon)$ are the objective parameters to judge the performance of denoising, which are calculated as follows:

$\mathrm{SNR}=10 \log _{10}\left\{\frac{\sum_{i=1}^{M}\left[y(i)-\mu_{y}\right]^{2}}{\sum_{i=1}^{M}\left[n(i)-\mu_{n}\right]^{2}}\right\}$,

NRMS $=\sqrt{\frac{\sum_{i=1}^{M}[n(i)]^{2}}{M}}$,

$\varepsilon=100 \times \frac{\rho_{1}-\rho_{0}}{\rho_{0}}$,

$i(t)=u_{i}(t) / R_{\mathrm{S}}$

where $\mu_{y}$ and $\mu_{n}$ denote the mean values of the useful signal and the noise separately. $y(i)$ and $n(i)$ are the useful signal and the noise separately, $M$ is the length, $\rho_{0}$ denotes the complex resistivity calculated without noise, and $\rho_{1}$ is the complex resistivity calculated with the noise added to $\rho_{0} . R_{\mathrm{S}}$ is the value of the sampling resistor $\left(R_{\mathrm{S}}=1 \Omega\right)$, and $u_{i}(t)$ is the voltage at the sampling resistor.

To validate the effectiveness of the ECI system, we performed a resistance-capacitance experiment, as shown in Fig. 5. The circuit parameters are chosen to be $R_{\mathrm{A}}=30.3 \Omega / 5 \mathrm{~W}, R_{\mathrm{MN}}=30.1 \Omega / 5 \mathrm{~W}, R_{\mathrm{B}}=30 \Omega / 5 \mathrm{~W}$, and $C_{\mathrm{MN}}=470 \mu \mathrm{F}$. We recorded the applied voltage $u_{T}(t)$, the injected current $i(t)$, and the measured potential signal $u(t)$ as the raw signals. These signals are a three-order spread spectrum pseudorandom sequence at the clock cycle of $0.0125 \mathrm{~s}$, as shown in Fig. 6a-c and Table 1.

Since our experiment is in a stable environment, we consider the system to be linear time-invariant, and the noise from the current and voltage measurements are linearly superpositioned (Pelton and Sill, 1983; Vinegar and Waxman, 1984; Garrouch and Sharma, 1998). Therefore, it is actually equivalent whenever the noise is added to the injected current
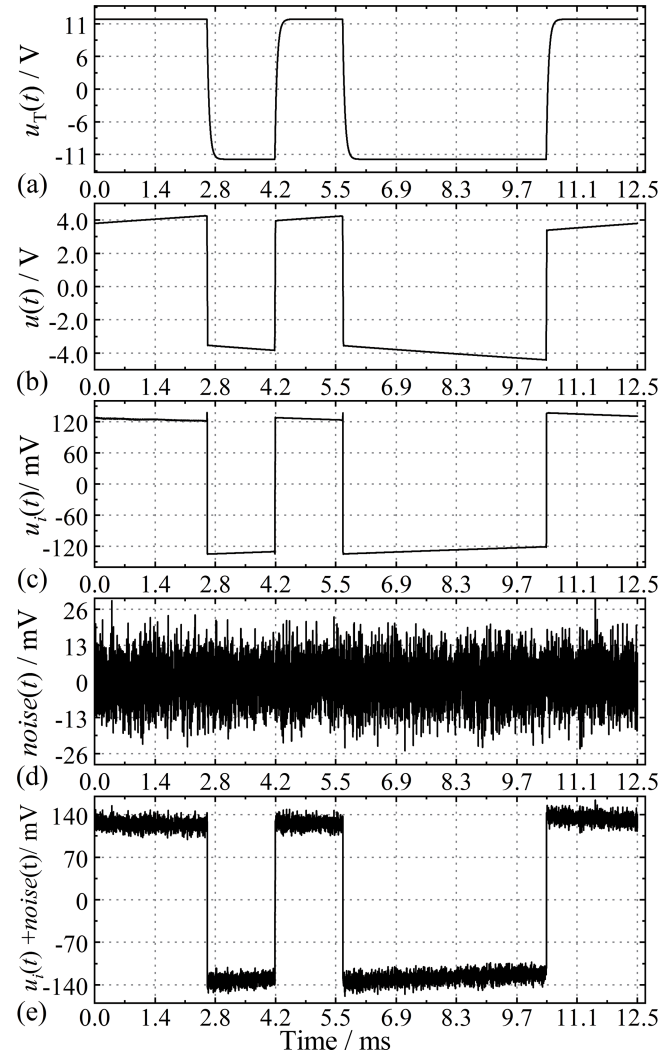

Figure 6. The time waves of (a) the applied voltage $u_{T}(t)$, (b) the measured potential signal $u(t)$, (c) the voltage $u_{i}(t)$ at the sampling resistor, (d) noise $(t)$, and (e) the synthetic signal $\left(u_{i}(t)+\right.$ noise $\left.(t)\right)$.

Table 1. Amplitude and phase values of complex resistivity obtained with Fig. 6a-c.

\begin{tabular}{lrrrr}
\hline $\begin{array}{l}\text { Frequency } \\
(\mathrm{Hz})\end{array}$ & $\begin{array}{r}\text { Theoretical } \\
\text { amplitude } \\
(\Omega)\end{array}$ & $\begin{array}{r}\text { Theoretical } \\
\text { phase } \\
(\mathrm{rad})\end{array}$ & $\begin{array}{r}\text { Measured } \\
\text { amplitude } \\
(\Omega)\end{array}$ & $\begin{array}{r}\text { Measured } \\
\text { phase } \\
(\mathrm{rad})\end{array}$ \\
\hline 80.2 & 30.8 & -0.14 & 30.8 & -0.14 \\
160.4 & 30.4 & -0.07 & 30.3 & -0.08 \\
320.8 & 30.2 & -0.03 & 30.7 & -0.03 \\
\hline
\end{tabular}



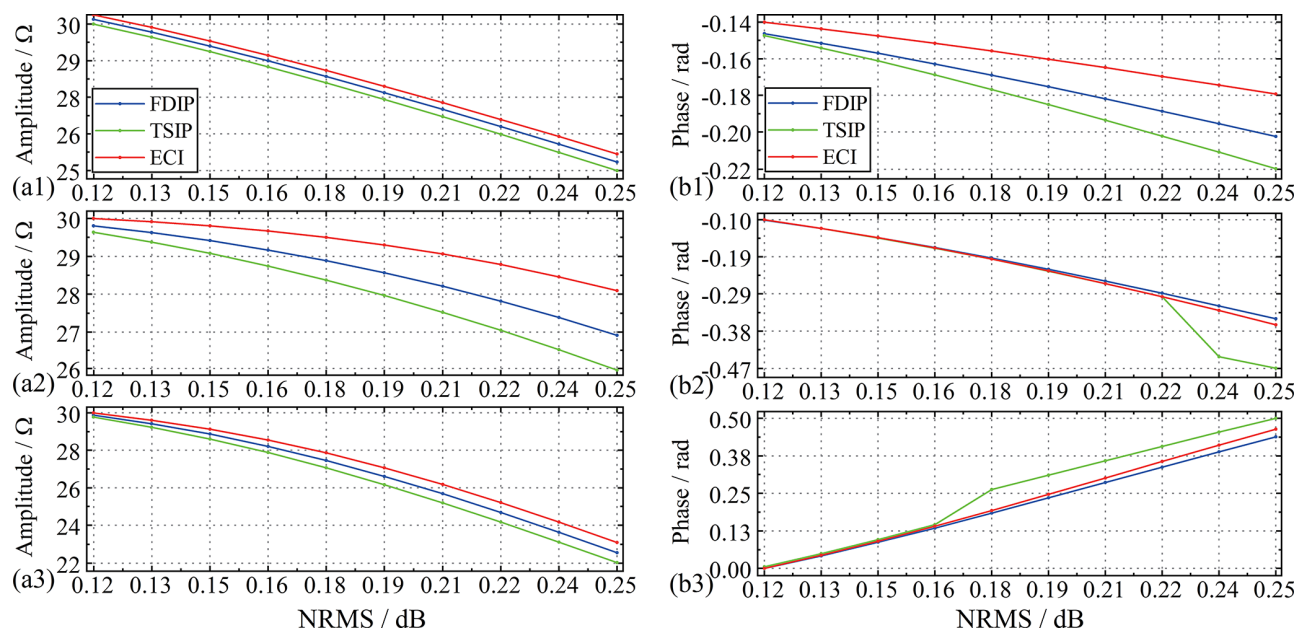

Figure 7. Amplitude and phase of complex resistivity values at $(\mathbf{a 1}, \mathbf{b 1}) 80 \mathrm{~Hz}$, and (a2, b2) $160 \mathrm{~Hz},(\mathbf{a} 3, \mathbf{b 3}) 320 \mathrm{~Hz}$ using the three methods.

$i(t)$, the measured potential signal $u(t)$, or the applied voltage $u_{T}(t)$. Therefore, the injected current $i(t)$ is only polluted by the synthetic background noise, including Gaussian and impulsive, as shown in Fig. 6d and e. Thirdly, the complex resistivity of the main frequency is considered and discussed because the main energy of the pseudorandom signal is concentrated on the main frequency $(\mathrm{He}, 2017)$. Finally, for detailed comparisons between the ECI algorithm and the others, we add the synthetic Gaussian and impulsive noises to the response signal $i(t)$, respectively.

We use synthetic Gaussian noise with the deviation and mean values of 0.1 and 1.1 as a standard template. The excitation signal $i(t)$ is polluted by synthetic different energy levels of the Gaussian noise. Figure 7 shows that the denoised results are obtained and compared at the three main frequencies when the NRMS ranges from 0.12 to 0.25 . The figure shows that as the NRMS increases, the complex resistivity information obtained by each algorithm decreases. However, the amplitude spectrum after ECI processing has the slowestfalling speed, and the phase spectrum has the slowest-falling speed at $80 \mathrm{~Hz}$.

Previous literature has shown that if the percentages of outliers in impulsive noise exceed $50 \%$, the traditional denoising algorithm will be limited (Liu and Chen, 2016; Liu et al., 2017a). Thus, synthetic impulsive noise is added to the excitation signal $i(t)$ in $10 \%$ steps. Their standard deviations (SDs) and skewness values (SKs) are shown in Fig. 8. As depicted in Fig. 9, the three algorithms have a certain degree of denoising performance versus the different percentages of the synthetic outliers against the raw data. The figure shows that with the discrete points of impulse noise growing, the NRMS is different. The amplitude spectrum and phase spectrum of complex resistivity obtained by each algorithm fluctuate. The amplitude spectrum after ECI processing remained the slowest-falling speed. Although the noise reduction performance of the phase spectrum processed by ECI

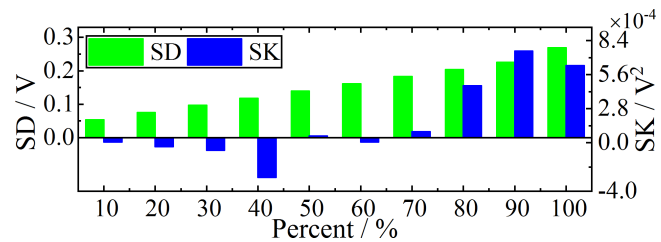

Figure 8. The standard deviations (SDs) and skewness values (SKs) of synthetic impulsive noise.

does not stand out, the overall change of the amplitude spectrum after ECI processing is still slow, especially when the discrete point is more than $60 \%$.

\section{Experiment on real SSIP data record}

To further verify the performance of the ECI algorithm, the Wenner array, which is the traditionally applied system in the field, was selected for performing laboratory tests, as shown in Figs. 10 and 11. SSIP data was acquired with a high-density meter and 20 electrodes at $1 \mathrm{~m}$ spacing. A Wenner acquisition sequence was adopted with 55 potential measurements expressed using the green points. The figure shows an example of two high-resistance cavities. The two cavities were presented by the letters $\mathrm{A}$ and $\mathrm{B}$, and their calibers were about $1.8 \mathrm{~m} \times 2 \mathrm{~m}$. The two cavities are buried by loess. The loess is measured to have an electronic resistivity of $36 \Omega \mathrm{m}$. The measured excitation signal had a range between 0.04 and $0.19 \mathrm{~A}$ approximately. The transmitter output signal is a three-order sequence with $80 \mathrm{~Hz}$ frequency, and its voltage is about $\pm 11.8 \mathrm{~V}$. The sampling frequency is $625 \mathrm{kHz}$. The excitation and response data of 40 periods were recorded at each point.

Figure 12 demonstrates the experimental SSIP data processed by the three algorithms, inverted with Res2DInv (Ar- 

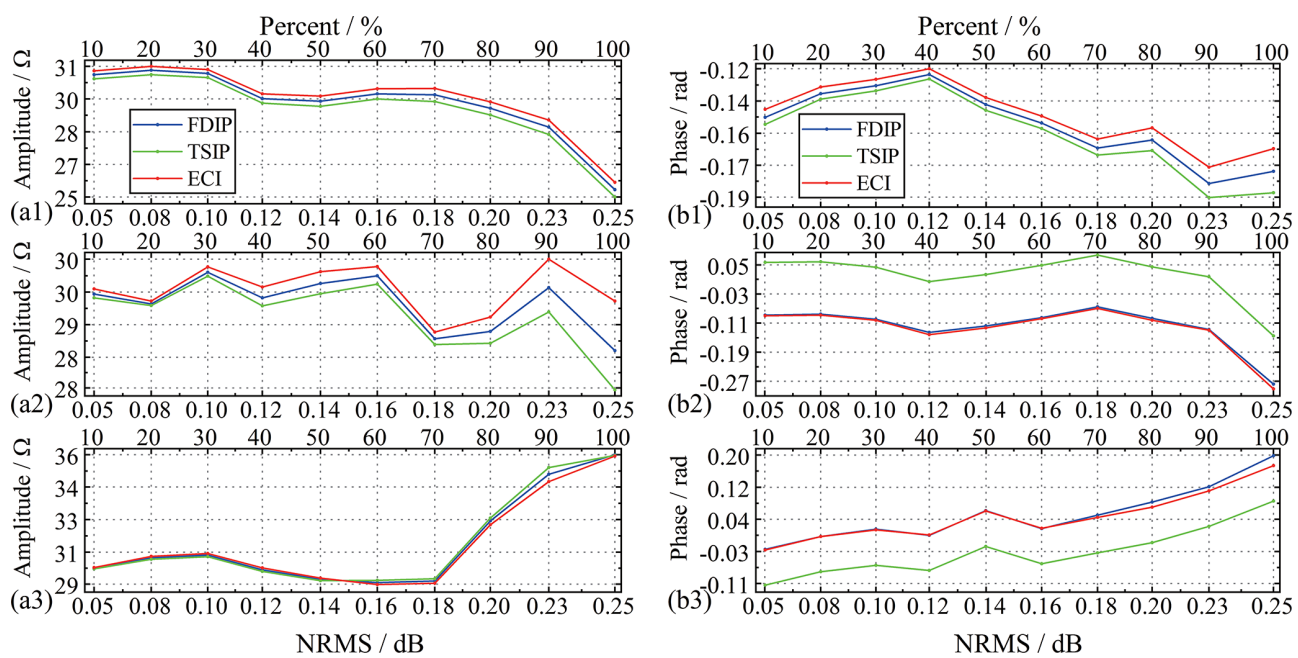

Figure 9. Complex resistivity values at (a1, b1) $80 \mathrm{~Hz},(\mathbf{a} 2, \mathbf{b 2}) 160 \mathrm{~Hz}$, and (a3, b3) $320 \mathrm{~Hz}$ using the three methods.
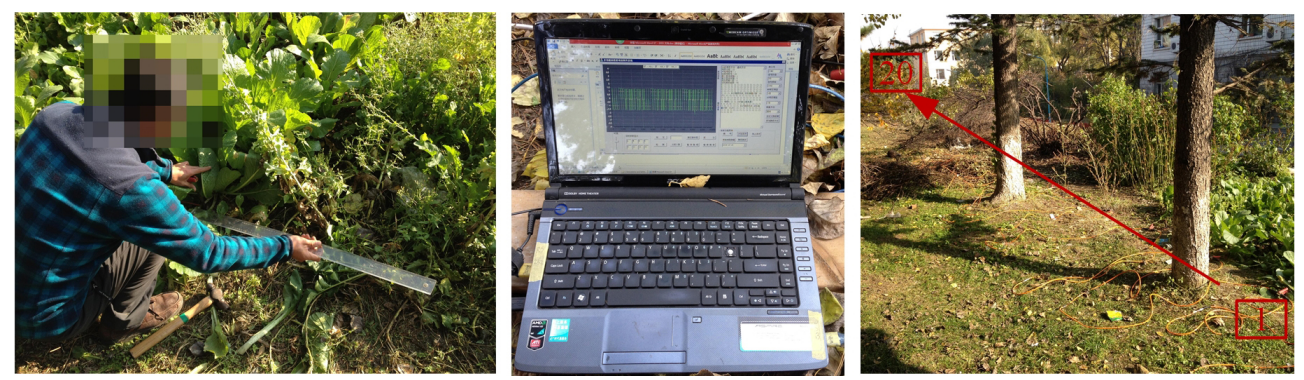

Figure 10. Diagram of the field test.

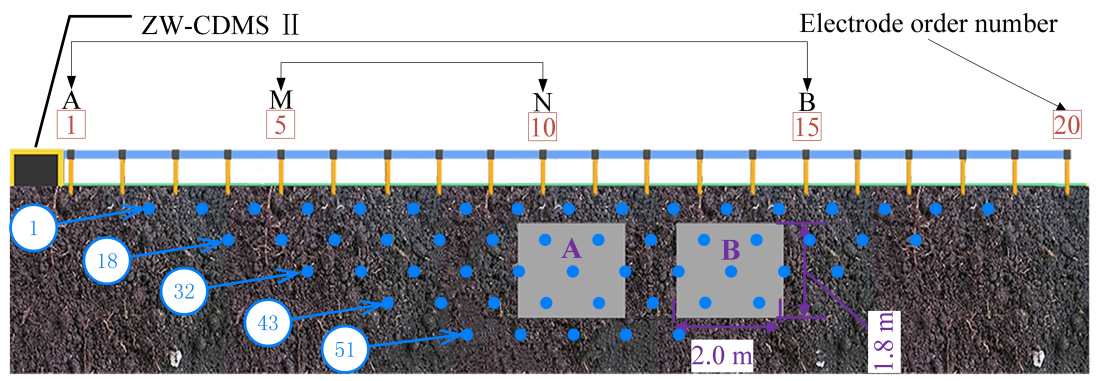

Figure 11. The schematic of the two high-resistance cavities.

ifin et al., 2019). It can be observed that the location and shape of two abnormal bodies are distinguished only in the ECI algorithm while recognized as one whole in the other algorithms. We believe the reason that ECI has higher detection precision is due to its higher denoising ability.

To verify the reason for the improved detecting precision, the SDs of data points are calculated from 18 to 50 (Fig. 11), as shown in Fig. 13. This figure shows that the 33 SD in ECI processing the SSIP data is the lowest at all points. The average SD values in ECI processing of the SSIP data are $7 \%$ and $3.8 \%$ lower than the FDIP and TSIP methods, respectively. Also, the maximum value of SDs with the ECI method is $5 \%$ and $1.4 \%$ lower than the others, and the minimum value is $8 \%$ and $10 \%$ lower, respectively.

Meanwhile, amplitude-frequency $|\rho(f)|$ and phasefrequency $\varphi(f)$ characteristics of complex resistivity are calculated by the three algorithms (one period) in survey point no. 38 in Fig. 11.

For example, Fig. 14a1 and a 2 show that the amplitude and phase of the complex resistivity spectrum for this point at $80 \mathrm{~Hz}$ processed by FDIP are $39.7 \Omega \mathrm{m}$ and $-0.0881 \mathrm{rad}$, the amplitude and phase are $40.9 \Omega \mathrm{m}$ and $6.12 \mathrm{rad}$ when at $160 \mathrm{~Hz}$, and the amplitude and phase are $38.7 \Omega \mathrm{m}$ and $-0.253 \mathrm{rad}$ when at $320 \mathrm{~Hz}$. As depicted in Fig. 14, the 

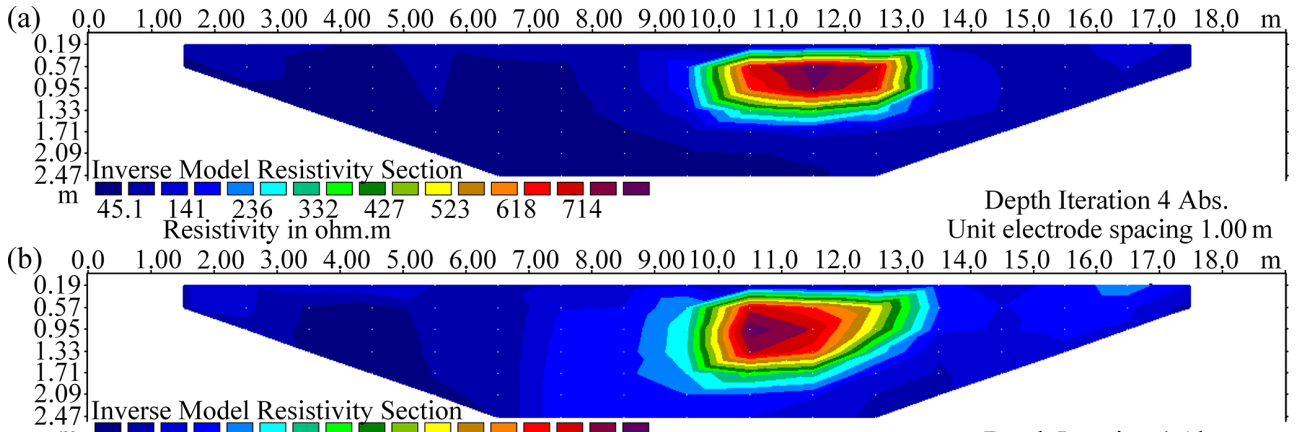

m $56.3 \quad 150 \quad 244338 \quad 433 \quad 526 \quad 621714$ Depth Iteration 4 Abs.

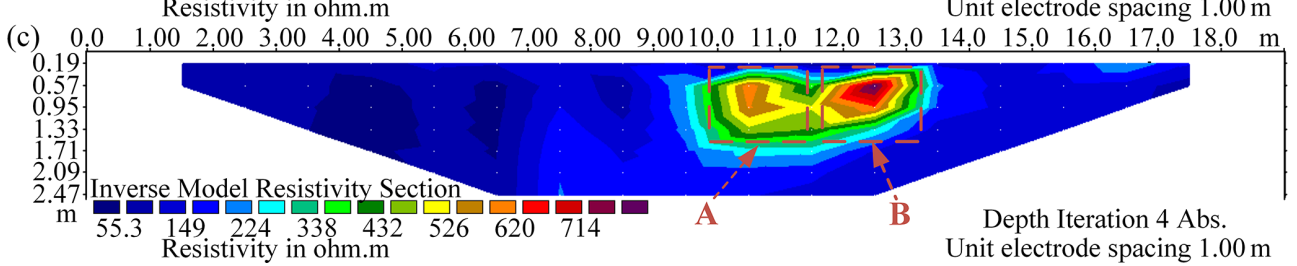

Figure 12. Inverted resistivity sections of the two high-resistivity anomalies (A and B) at $80 \mathrm{~Hz}$ with using (a) the FDIP method, (b) the TSIP algorithm, and (c) the ECI algorithm.

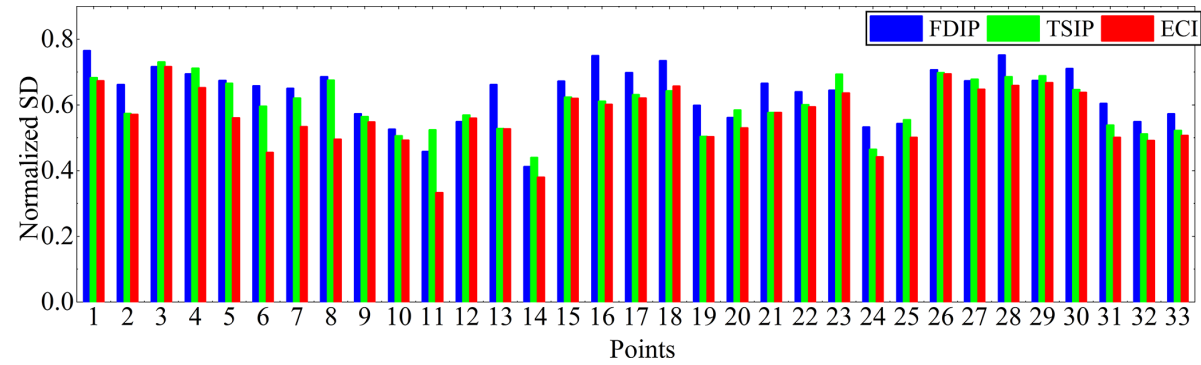

Figure 13. Standard deviation (SD) values of the ECI algorithm and the others compared to the data dots from 18 to 50 at $80 \mathrm{~Hz}$.

complex resistivity processed by the ECI shows a linear trend with the three main frequencies. Also, the SD of the amplitude-frequency $|\rho(f)|$ characteristic is 0.10 and 0.49 lower than the others, and the SD of the phase-frequency $\varphi(f)$ is 3.56 and 0.03 lower. Therefore, we believe that the ECI algorithm has an advantage in suppressing background noise, which benefits the subsequent steps in SSIP data processing and imaging.

\section{Discussion}

The simulation results indicate that the ECI algorithm has very good performance in noise reduction and robustness. Along with the increase of the Gaussian noise level, we found that the ECI algorithm can, to some extend, overcome the shortcomings of the TSIP algorithm has, i.e., being susceptible to the noise of the current. This result coincided with Eqs. (6) and (13), which provides a novel approach for correlated identification noise reduction. In the impulsive noise experiment, we found that the ECI algorithm still has good noise reduction when the discrete point is more than $60 \%$, which compensates for the disadvantage of the traditional denoising algorithm. Moreover, these simulation results also reveal that the ECI algorithm should have high robustness.

The standard deviation analysis of the real data indicates that the ECI algorithm improves the accuracy and robustness of the collected data, which are compatible with the simulation analyses. This consistency shows that the ECI algorithm can obtain the location and shape of two abnormal bodies by improving the SNR of SSIP data, which can increase the resolution of inversion results.

\section{Conclusions}

We propose the ECI algorithm that effectively attenuates the background noise in SSIP data and improves the complex resistivity spectrum. This algorithm uses the correlation function to neutralize the influence of the background noise in the SSIP data, and the spectrum complex resistivity can be calculated at multiple frequencies by the formula of the complex 

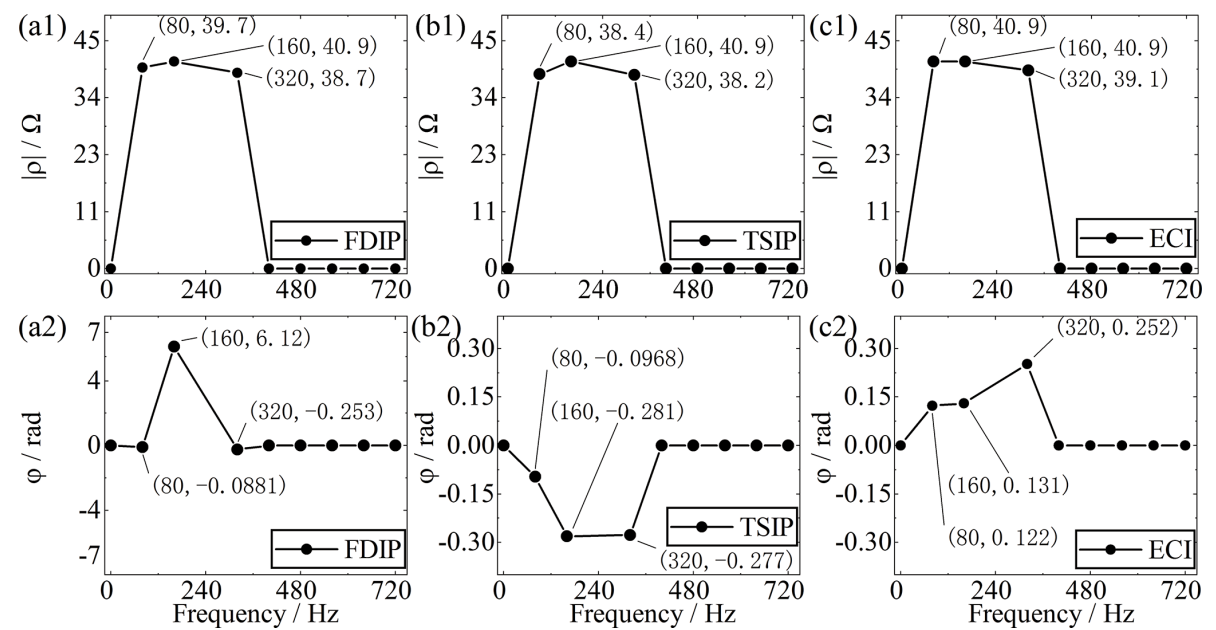

Figure 14. Complex resistivity spectrum calculated by the three algorithms (one period) in survey point no. 38 .

resistivity. Simulation results show that the ECI algorithm can effectively attenuate the background noise and improve the SNR. Subsequently, the practicability of the ECI algorithm is further verified by a field test. The results demonstrate that the SD of the SSIP data is improved, which benefits the accuracy and stability of the collected data. There is a good agreement between the complex resistivity and the geological target body with high resistance, which indicates that the ECI algorithm can help to improve the quality of interpretation and inversion in the survey area. For the amplitude spectrum, the ECI algorithm can more effectively suppress the background noise, including the Gaussian random and impulsive noises. Still, its effect is very limited for the phase spectrum. Therefore, a denoising algorithm based on pseudorandom sequence correlation identification is still left open for more investigation.

Code availability. The code is a collection of routines in MATLAB (MathWorks) and is available upon request to the author (e-mail: hsmfly1982@163.com).

Data availability. All the SSIP data are collected by the ZWCMDSII and are available upon request to the author (e-mail: hsmfly1982@163.com).

Author contributions. SH and YW designed the study, performed the research, analyzed data, and wrote the paper. JG contributed to language polishing and response. XJ and HX contributed to refining the ideas, carrying out additional analyses, and finalizing this paper.

Competing interests. The authors declare that they have no conflict of interest.
Acknowledgements. We are grateful for the help of Jun Wang, Shi Zhu, Hui Wang, and Jinshi Cui. We thank the editors and the reviewers for the constructive comments that helped to improve this article.

Financial support. This research has been supported by the Key Technology Projects of Science and Technology Department of Jilin Province Scientific (grant no. 20190303015SF), a research project of Jilin Provincial Department of Education (grant nos. JJKH20210692KJ and JJKH20211053KJ), and the Fundamental Research and Theme Funds for Changchun Institute of Technology, China.

Review statement. This paper was edited by Richard Gloaguen and reviewed by three anonymous referees.

\section{References}

Arifin, M. H., Kayode, J. S., Izwan, M. K., Zaid, H. A. H., and Hussin, H.: Data for the potential gold mineralization mapping with the applications of Electrical Resistivity Imaging and Induced Polarization geophysical surveys, Data in Brief, 22, 830835, https://doi.org/10.1016/j.dib.2018.12.086, 2019.

Chen, R. J., Luo, W. B., and He, J. S.: High precision multi-frequency multi-function receiver for electrical exploration, 2007 8th International Conference on Electronic Measurement and Instruments (ICEMI'07), 16-18 August 2007, Xian, China, IEEE, Expanded Abstracts, 599-602, https://doi.org/10.1109/icemi.2007.4350521, 2007.

Collett, L. S., Brant, A. A., Bell, W. E., Ruddock, K. A., Seigel, H. O., and Wait, J. R.: Laboratory investigation of overvoltage, Overvoltage research and geophysical applications, International series of monographs on earth sciences, Pergamon, New York, USA, 50-70, https://doi.org/10.1016/b978-0-08-009272$0.50009-1,1959$. 
Garrouch, A. A. and Sharma M. M.: Dielectric dispersion of partially saturated porous media in the frequency range $10 \mathrm{~Hz}$ to $10 \mathrm{MHz}$, The Log Analyst, 39, 48-53, 1998.

Guo, H.: Study of key technology and data fusion of multi-probe penetration based on gas hydrate exploration, $\mathrm{PhD}$ Thesis, China University of Geosciences, Wuhan, China, 2017.

Han, S. L., Zhang, S. G., Liu, J. X., Hu, J., and Zhang, W. S.: Integrated interpretation of dual frequency induced polarization measurement based on wavelet analysis and metal factor methods, T. Nonferr. Metal. Soc., 23, 1465-1471, https://doi.org/10.1016/S1003-6326(13)62618-7, 2013.

He, G.: Wide area electromagnetic method and pseudo random signal method, Higher Education Press, Beijing, China,, 2017.

He, G., Wang, J., Zhang, B. Y., Li, M., and Ma, C.: Design of High-density Electrical Method Data Acquisition System, Instrument Technique and Sensor, 8, 18-19, https://doi.org/10.3969/j.issn.1002-1841.2014.08.007, 2014.

He, J. H., Yang, Y., Li, D. Q., and Weng, J. B.: Wide field electromagnetic sounding methods, in: Symposium on the Application of Geophysics to Engineering and Environmental Problems (SAGEEP 2015), 22-26 March 2015, Texas, USA, EEGS, Expanded Abstracts, 325-329, https://doi.org/10.4133/sageep.28047, 2015

He, J. S.: Dual-frequency IP, T. Nonferr. Metal. Soc., 3, 1-10, 1993.

Høyer, A. S., Klint, K. E. S., Fiandaca, G., Maurya, P. K., Christiansen, A. V., Balbarini, N., Bjerg, P. L., Hansen, T. B., and Møller, I.: Development of a high-resolution 3D geological model for landfill leachate risk assessment, Eng. Geol., 249, 4559, https://doi.org/10.1016/j.enggeo.2018.12.015, 2018.

Kimiaefar, R., Siahkoohi, S. H., Hajian, A., and Kalhor, A.: Random noise attenuation by Wiener-ANFIS filtering, J. Appl. Geophys., 159, 453-459, https://doi.org/10.1016/j.jappgeo.2018.05.017, 2018.

Li, G., Liu, X., Tang, J., Li, J., Ren, Z., and Chen, C.: De-noising low-frequency magnetotelluric data using mathematical morphology filtering and sparse representation, J. Appl. Geophys., 172, 103919, https://doi.org/10.1016/j.jappgeo.2019.103919, 2019.

Li, M., Wei, W., Luo, W., and Xu, Q: Time-domain spectral induced polarization based on pseudo-random sequence, Pure Appl. Geophys., 170, 2257-2262, https://doi.org/10.1007/s00024-0120624-z, 2013.

Liu, N.: Preprocessing and Research of denosing methods for marine controlled source electromangnetic data, MSc Thesis, Jilin University, Jilin, China, 2015.

Liu, W. Q. and Chen, R. J.: Coherence analysis for multi-frequency induced polarization signal processingin strong interference environment, The Chinese Journal of Nonferrous Metals, 26, 655665, https://doi.org/10.19476/j.ysxb.1004.0609.2016.03.022, 2016 (in Chinese).

Liu, W. Q., Chen, R. J., Cai, H. Z., and Luo, W. B.: Robust statistical methods for impulse noise suppressing of spread spectrum induced polarization data, with application to a mine site, Gansu province, China, J. Appl. Geophys., 135, 397-407, https://doi.org/10.1016/j.jappgeo.2016.04.020, 2016.

Liu, W. Q., Chen, R. J., Cai, H. Z., Luo, W. B., and Revil, André: Correlation analysis for spread spectrum induced polarization signal processing in electromagnetically noisy environments,
Geophysics, 82, E243-E256, https://doi.org/10.1190/geo20160109.1, 2017a.

Liu, W. Q., Wang, J. L., and Lin, P. R.: Signal processing approaches to obtain complex resistivity and phase at multiple frequencies for the electrical exploration method, B. Geofis. Teor. Appl., 58, 103-114, https://doi.org/10.4430/bgta0190, 2017b.

Liu, W. Q., Lü, Q. T., Chen, R. J., Lin, P. R., Chen, C. J., Yang, L. Y., and Cai, H. Z.: A modified empirical mode decomposition method for multiperiod time-series detrending and the application in full-waveform induced polarization data, Geophys. J. Int., 217, 1058-1079, https://doi.org/10.1093/gji/ggz067, 2019.

Lu, Q. T., Zhang, X. P., Tang, J. T., Jin, S., Liang, L. Z., Wang, X. B., Lin, P. R., Yao, C. L., Gao, W. 1., Gu, J. S., Han, L. G., Cai, Y. Z., Zhang, J. C., Liu, B. L., and Zhao, J. H.: Review on advancement in technology and equipment of geophysical exploration for metallic deposits in china, Chinese J. Geophys., 62, 3629-3664, https://doi.org/10.6038/cjg2019N0056, 2019 (in Chinese).

Meng, Q. X., Pan, H. P., and Luo, M.: A study on the discrete image method for calculation of transient electromagnetic fields in geological media, Appl. Geophys., 12, 493-502, https://doi.org/10.1007/s11770-015-0517-x, 2015.

Mo, D., Jiang, Q. Y., Li, D. Q., Chen, C. J., Zhang, B. M., and Liu, J. W.: Controlled-source electromagnetic data processing based on gray system theory and robust estimation, Appl. Geophys., 14, 570-580, https://doi.org/10.1007/s11770-017-0646-5, 2017.

Neelamani, R., Baumstein, A. I., Gillard, D. G., Hadidi, M. T., and Soroka, W. L.: Coherent and random noise attenuation using the curvelet transform, The Leading Edge, 27, 240-248, https://doi.org/10.1190/1.2840373, 2008.

Pelton, W. H. and Sill, W. R.: Interpretation of complex resistivity and dielectric data, Geophysical Transactions, 29, 297-330, 1983.

Revil, A., Karaoulis, M., Johnson, T., and Kemna, A.: Review: Some low-frequency electrical methods for subsurface characterization and monitoring in hydrogeology, Hydrogeol. J., 20, $617-$ 658, https://doi.org/10.1007/s10040-011-0819-x 2012.

Revil, A., Razdan, M., Julien, S., Coperey, A., Abdulsamad, F., Ghorbani, A., and Rossi, M.: Induced polarization response of porous media with metallic particles - Part 9: Influence of permafrost, Geophysics, 84, E337-E355, https://doi.org/10.1190/geo2019-0013.1, 2019.

Seigel, H. O.: Mathematical formulation and type curves for induced polarization, Geophysics, 24, 547-565, https://doi.org/10.1190/1.1438625, 1959.

Vinegar, H. J. and Waxman, M. H.: Induced polarization of shaly sands, Geophysics, 49, 1267-1287, https://https://doi.org/10.1190/1.1441755, 1984.

Wang, Y. B. and He, J. S.: A hybrid coding and its application to the oil and gas fracturing intelligent real time monitoring system based on pseudorandom signal, Geophysical and Geochemical Exploration, 44, 74-80, https://doi.org/10.11720/wtyht.2020.2288, 2020.

Wait, J. R.: The variable-frequency method, Overvoltage research and geophysical applications, International series of monographs on earth sciences, Pergamon, 29-49, https://doi.org/10.1016/b978-0-08-009272-0.50008-x, 1959.

Xi, X. L., Yang, H. C., He, L. F., and Chen, R. J.: Chromite mapping using induced polarization method based on spread spectrum technology, Symposium on the Application of Geophysics to 
Engineering and Environmental Problems (SAGEEP 2013), 1721 March 2013, Denver, Colorado, USA, EEGS, Expanded Abstracts, 13-19, https://doi.org/10.4133/sageep2013-015.1, 2013.

Xi, X. L., Yang, H. C., Zhao, X. F., Yao, H. C., Qiu, J. T., Shen, R. J., and Chen, R. J.: Large-scale distributed 2D/3D FDIP system based on ZigBee network and GPS, Symposium on the Application of Geophysics to Engineering and Environmental Problems (SAGEEP 2014), 16-20 March 2014, Boston, Massachusetts, USA, EEGS, Expanded Abstracts, 130-139, https://doi.org/10.1190/SAGEEP.27-055, 2014.

Zhang, B. Y., He, G., and Wang J.: New High-density Electrical Instrument Measuring System, Instrument Technique and Sensor, 1, 24-26, https://doi.org/10.3969/j.issn.1002-1841.2014.01.009, 2014.
Zhang, Q. D., Hao, K. X., and Li, M.: Improved correlation identification of subsurface using all phase FFT algorithm, KSII Transactions on Internet \& Information Systems, 14, 495-513, https://doi.org/10.3837/tiis.2020.02.002, 2020.

Zonge, K. L. and Wynn, J. C.: Recent advances and applications in complex resistivity measurements, Geophysics, 40, 851-864, https://doi.org/10.1190/1.1440572, 1975. 